\title{
A importância do aconselhamento no exame rápido de HIV em gestantes durante o pré-natal
}

\section{The importance of counseling in rapid HIV testing for pregnant women at prenatal care}

\author{
Sabrina Monique Previati ${ }^{1,2}$ (D), Daniel Malingre Vieira ${ }^{3}$ (D), Márcia Barbieri ${ }^{4,5}$ (D)
}

1. Unidade de Saúde da Mulher (USM) em São Vicente, São Vicente, SP, Brasil.2. Pós-graduanda em Saúde Coletiva pela Universidade Nove de Julho (UNINOVE), São Paulo, SP, Brasil. 3. Pós-graduando em Gestão Pública Municipal pela Universidade Federal de São Paulo (UNIFESP), Santos, SP, Brasil. 4. Docente do Departamento de Enfermagem na Saúde da Mulher da Universidade Federal de São Paulo (UNIFESP), São Paulo, SP, Brasil. 5. Docente do Programa de Pós-Graduação em Enfermagem da Universidade Federal de São Paulo (USP), São Paulo, SP, Brasil

\section{Resumo}

Introdução: o aumento do número de casos de gestantes infectadas com o HIV é preocupante, entre as estratégias fomentadas para redução da transmissão vertical o aconselhamento pré-teste rápido de HIV apresenta-se como importante medida preventiva. Objetivo: Demonstrar, por meio de revisão da literatura, a importância do aconselhamento no teste rápido de HIV em gestantes. Método: estudo descritivo com pesquisa de revisão bibliográfica realizado por meio de artigos e publicações científicas encontradas nas bases de dados SCIELO, LILACS, CAPES e PUBMED com abrangência temporal de 2004 a 2018, utilizando-se os seguintes descritores: HIV, Aconselhamento, Teste rápido, Gestantes, Pré-Natal, em português e inglês. Resultados: obtiveram-se 18 artigos relacionados ao tema, utilizados após criteriosa seleção em revisão bibliográfica integrativa que foram utilizados como base teórica para a discussão acerca do tema do trabalho. Conclusão: observou-se que, além da necessidade em agilizar o diagnóstico, deve-se também fornecer as orientações necessárias pré e pós teste das gestantes atendidas para que o atendimento as esclareça de modo a priorizar medidas profiláticas na prevenção da doença e, no caso de positividade do resultado do exame, reduzir a transmissão vertical e estimular o seguimento do tratamento da condição patológica, além de estabelecer vínculos com o paciente visando à promoção de seu autocuidado.

Palavras-chave: HIV. Gravidez. Teste rápido. Aconselhamento. Pré-natal.

\begin{abstract}
Introduction: The increase in the number of cases of pregnant women infected with HIV is a concern, among the strategies promoted to reduce vertical transmission, rapid pre-test HIV counseling is an important preventive measure. Objective: to demonstrate, through a review of the literature, the importance of counseling in the rapid test of HIV in pregnant women. Method: a descriptive study with bibliographic review research carried out through articles and scientific publications found in the SCIELO, LILACS, CAPES and PUBMED databases with temporal coverage from 2004 to 2018, using the following descriptors: HIV, Counseling, Rapid Testing, Pregnant women, Pre-Natal in Portuguese and English. Results: there were 18 articles related to the topic used after careful selection in an integrative bibliographical review that were used as a theoretical basis for the discussion about the theme of the work. Conclusion: it was observed that in addition to the need to speed up the diagnosis, it is also necessary to provide the necessary pre and post-test guidelines of the pregnant women attended so that care clarifies them in order to prioritize prophylactic measures in the prevention of the disease and, in the case of positivity of the result of the examination, reduce vertical transmission and stimulate follow-up of the treatment of the pathological condition, besides establishing bonds with the patient aiming to promote his self-care.
\end{abstract}

Key words: HIV. Pregnancy. Rapid testing. Counseling. Prenatal.

\section{INTRODUÇÃO}

Desde o início da epidemia, em 1980, até junho de 2017, o Brasil teve 882.810 casos registrados de AIDS, condição em que a doença já se manifestou, de acordo com o último boletim epidemiológico. Foram 18,5 casos a cada 100.000 habitantes no Brasil, registrados em 2016 ${ }^{1}$. Entre junho de 2000 até junho de 2017 houve notificação de 108.134 casos envolvendo gestantes infectadas com HIV².

A taxa de detecção de gestantes com HIV no Brasil vem apresentando uma pequena tendência de aumento nos últimos anos, em grande parte devido ao grande incremento de testes rápidos distribuídos pela Rede Cegonha. Em 2012, foram distribuídos 366.910 testes de HIV para gestantes, enquanto em 2017, somente até o mês de outubro, já haviam sido distribuídos 3.350 .440 testes.

Em um período de dez anos, houve aumento de $23,8 \%$ na taxa de detecção de HIV em gestantes; em 2006, a taxa observada foi de 2,1 casos/mil nascidos vivos e, em 2016, passou para 2,6/mil nascidos vivos. A tendência de crescimento também é verificada em todas as regiões do Brasil, exceto na região Sudeste, com taxa de 2,2 casos/mil nascidos vivos em 2006 e 2,0 em 2016. As regiões Norte e Nordeste foram as que apresentaram maiores elevações na taxa; ambas apresentavam taxa de 1,2 em 2006, 
passando para 2,9 e 2,0 casos/mil nascidos vivos em 2016, respectivamente. Em 2016, a região Sul mostrou a maior taxa de detecção $\left(5,6\right.$ casos/mil nascidos vivos) ${ }^{1}$.

A epidemia de AIDS é um problema de grande magnitude que progride em todas as regiões do planeta. Ultimamente, dados epidemiológicos registram aumento de casos em mulheres, representando a possibilidade de aumento da transmissão vertical do $\mathrm{HIV}^{3}$.

O sucesso da prevenção da transmissão vertical depende da identificação da totalidade das gestantes infectadas e de que essa detecção seja a mais precoce possível ${ }^{4,5}$.

Uma atenção pré-natal e puerperal qualificada e humanizada ocorre por meio da incorporação de condutas acolhedoras e sem intervenções desnecessárias e do fácil acesso a serviços de saúde de qualidade, com ações que integrem todos os níveis da atenção: promoção, prevenção e assistência à saúde da gestante e do recém-nascido ${ }^{6}$. 0 governo brasileiro vem-se empenhando para reduzir as taxas de transmissão vertical do HIV no país.

Entre as medidas, há o aconselhamento e a realização de sorologia para HIV no pré-natal ${ }^{5}$. Estudos pertencentes à revisão bibliográfica realizada em $2017^{6}$ referem redução na transmissão vertical do HIV como principal benefício da adesão da gestante soropositiva aos cuidados no pré-natal, parto e puerpério.

No Brasil, a partir de 1989, as estratégias de testagem e aconselhamento passaram a ocupar lugar de destaque nos programas de prevenção. $O$ aconselhamento se inseriu nas ações propostas pelo programa nacional da AIDS, pautado na lógica do pré e do pós-teste anti-HIV que, até então, tinha abordagem voltada para o suporte emocional e a aceitação da doença. $\mathrm{O}$ aconselhamento, no entanto, transcende o âmbito da testagem e configura-se como uma ação em saúde, que contribui, potencialmente, para a redução da transmissão do HIV e de outras DST's, uma vez que promove a reflexão do indivíduo, tornando-o, sobretudo, sujeito no processo de prevenção e cuidado de $\mathrm{si}^{7}$.

O aconselhamento é entendido como um processo de escuta ativa individualizada e centrada no paciente. Ressalta-se que jovens entre 20 e 24 anos com escolaridade entre $5 a$ a 8 a série incompleta, representam $37,7 \%$ das gestantes infectadas com HIV ${ }^{1,8}$; portanto, é imprescindível, além de estabelecer relação de confiança entre os interlocutores, que haja comunicação adequada das informações prestadas, de segundo as particularidades de cada paciente objetivando ao melhor entendimento; dessa forma permitindo que o mesmo tenha possibilidade de reconhecer-se como sujeito de sua própria saúde e transformação ${ }^{8}$. $\mathrm{O}$ aconselhamento compreende alguns objetivos essenciais: o primeiro, de investir na prevenção da doença informar e abrir espaços para discutir a necessidade e as possibilidades de interferir no comportamento dos indivíduos, por exemplo, em relação ao uso de preservativos nas relações sexuais; o segundo, de garantir aos pacientes um espaço para estabelecer vínculos de confiança; os momentos de atendimento à mulher representam espaços oportunos para a realização do aconselhamento e para o acompanhamento e apoio às medidas preventivas. Um desses espaços importantes é o atendimento pré-natal ${ }^{9,10}$.

A lei do exercício profissional no 7.498/86 deixa claras as atribuições privativas do enfermeiro, cabendo-lhe educar visando à melhoria da saúde da população ${ }^{11}$.

Durante a minha experiência profissional na assistência às gestantes da baixada santista, pude observar que a maioria delas desconhece os modos de transmissão do HIV, janela sorológica, transmissão vertical e tratamento, expondo-se, assim, a situações de risco. $\mathrm{O}$ momento do aconselhamento pré-teste é de vital importância durante a abertura do pré-natal, pois é nela que se dedica maior tempo em razão da coleta de dados, anamnese e preenchimentos da documentação necessária para a abertura do pré-natal.

Nesse sentido, o presente trabalho tem como objetivo, por meio de revisão bibliográfica integrativa do tema, exaltar a importância do aconselhamento no teste rápido de HIV em gestantes.

\section{MÉTODOS}

Trata-se de um estudo descritivo com pesquisa de revisão bibliográfica integrativa focada em aconselhamento pré e pósteste rápido de HIV. A revisão bibliográfica integrativa consiste na análise crítica e detalhada de artigos anteriores selecionados sob certos critérios a respeito de determinado tema realizada por meio da leitura, verificação e revisão de publicações, livros e revistas relacionadas, que têm por objetivo realizar um levantamento do que há de atual sobre o assunto abordado. 0 principal objetivo da revisão integrativa é a integração entre a pesquisa científica e a prática profissional no âmbito da atuação profissional $^{12}$.

As buscas foram realizadas nos meses de julho e agosto de 2018 e foram encontradas publicações relativas ao tema abordado, utilizando os seguintes descritores, em conjunto, na pesquisa: HIV, Aconselhamento, Teste rápido, Gravidez e os keywords correspondentes em inglês: HIV, Counseling, Rapid test e Pregnancy a utilização dos descritores em conjunto foi adotada utilizando cada descritor entre aspas seguido de " $e$ " ou "and" para que a busca fosse referente a artigos que compreendessem todas as palavras chave juntas no mesmo artigo. As buscas foram realizadas em quatro bases de dados bibliográficas - SCIELO, PUBMED, LILACS e CAPES. A pré-seleção dos artigos encontrados constituiu-se através da leitura dos resumos disponíveis nas bases de dados sendo posteriormente descartados os artigos que não puderam ser visualizados na íntegra, foram selecionados por fim os artigos que se adequavam aos critérios de inclusão referentes ao objetivo e problemática 
do estudo. Em relação ao recorte temporal foram selecionados artigos publicados entre 2004 e 2018 que corresponde ao período que se enquadra os artigos selecionados na discussão do presente trabalho.

\section{RESULTADOS}

As bases de dados utilizadas na pesquisa (SCIELO, PUBMED, LILACS e CAPES) forneceram 101 resultados totais relativos à busca, os quais passaram por critério de seleção conforme descrito na quadro 1 abaixo. Dos resultados envolvendo a discussão a respeito do tema, foram selecionados 18 artigos para revisão bibliográfica integrativa, encontrados respectivamente na base de dados SCIELO (4 resultados totais, 3 selecionados); PUBMED, (68 resultados totais, 4 selecionados); LILACS (7 resultados totais, 2 selecionados) e CAPES, (22 resultados totais, 10 selecionados) (Quadro 1). Os principais achados encontramse resumidos no quadro 2 .

Quadro 1. Bases de dados, descritores utilizados, localização e seleção dos artigos.

\begin{tabular}{|clcc|}
\hline Base de Dados & Descritor & Artigos encontrados & $\begin{array}{c}\text { Artigos } \\
\text { selecionados }\end{array}$ \\
\hline SCIELO & HIV, Aconselhamento, Teste rápido e Gravidez & 4 & 3 \\
LILACS & HIV, Aconselhamento, Teste rápido e Gravidez & 7 & 2 \\
CAPES & HIV, Aconselhamento, Teste rápido e Gravidez & 22 & 10 \\
PUBMED & HIV, Counseling, Rapid test and Pregnancy & 68 & 4 \\
\hline
\end{tabular}

Quadro 2. Distribuição de artigos presentes na discussão e caracterização dos estudos.

\begin{tabular}{|c|c|c|c|c|}
\hline № & Título & Autores & Tipo de estudo & Recomendações/Conclusões \\
\hline 13 & $\begin{array}{l}\text { Atenção ao pré-natal de } \\
\text { baixo risco. }\end{array}$ & $\begin{array}{l}\text { MS.[Br]. Secretaria } \\
\text { de Atenção à Saúde } \\
\text { Departamento de } \\
\text { Atenção Básica }\end{array}$ & $\begin{array}{l}\text { Caderno de atenção básica/ } \\
\text { Manual de procedimentos } \\
\text { e normas técnicas. }\end{array}$ & $\begin{array}{l}\text { Os resultados confirmam a eficácia da } \\
\text { política de redução da transmissão vertical } \\
\text { do HIV instituída em nosso País. }\end{array}$ \\
\hline 14 & $\begin{array}{l}\text { Fatores associados à } \\
\text { submissão ao teste rápido } \\
\text { anti-HIV na assistência ao } \\
\text { parto. }\end{array}$ & $\begin{array}{l}\text { Oliveira MIC, Silva KS, } \\
\text { Gomes DM }\end{array}$ & Estudo transversal & $\begin{array}{l}\text { A cor não branca, a ausência de companheiro } \\
\text { e o elevado número de moradores na } \\
\text { residência foram mais submetidas ao teste } \\
\text { rápido anti-HIV, independente da realização } \\
\text { ou não de pré-natal o que demonstra } \\
\text { necessidade de qualificação das equipes de } \\
\text { saúde. }\end{array}$ \\
\hline 15 & $\begin{array}{l}\text { Coverage characterization } \\
\text { of prenatal in Maranhão } \\
\text { State, Brazil }\end{array}$ & $\begin{array}{l}\text { Costa GR, Chein MB, } \\
\text { Gama ME, Coelho LS, } \\
\text { Costa AS, Cunha CL, } \\
\text { Brito LM }\end{array}$ & $\begin{array}{l}\text { Estudo de } \begin{array}{r}\text { base } \\
\text { populacional, descritivo } \\
\text { quantitativo. }\end{array}\end{array}$ & $\begin{array}{l}\text { A cobertura do pré-natal foi de } 85,6 \% \text {; no } \\
\text { entanto, ao se considerar a cobertura de } \\
\text { pré-natal adequada, este foi de } 43,4 \% \text {, de } \\
\text { acordo com o preconizado pelo Ministério } \\
\text { da Saúde (Brasil). }\end{array}$ \\
\hline 16 & $\begin{array}{ll}\text { Cartografia } & \text { da } \\
\text { implementação do } & \text { do } \\
\text { teste rápido anti-HIV na } \\
\text { Estratégia Saúde da Família: } \\
\text { perspectiva de enfermeiros }\end{array}$ & $\begin{array}{l}\text { Silva TS, Nogueira CV, } \\
\text { Rosendo RAS }\end{array}$ & Pesquisa qualitativa. & $\begin{array}{l}\text { São necessários fornecimento adequado } \\
\text { de testes para as unidades, ampliação da } \\
\text { oferta do teste e expansão da capacitação } \\
\text { para outros membros da equipe da ESF. }\end{array}$ \\
\hline 17 & $\begin{array}{l}\text { O trabalho multiprofissional } \\
\text { na Estratégia Saúde da } \\
\text { Família: estudo sobre } \\
\text { modalidades de equipes. }\end{array}$ & $\begin{array}{l}\text { Pereira RCA, Rivera } \\
\text { FJU, Artmann E }\end{array}$ & $\begin{array}{l}\text { Pesquisa qualitativa do tipo } \\
\text { estudo de caso. }\end{array}$ & $\begin{array}{l}\text { O trabalho em equipe na ESF projeta } \\
\text { comunicação e cooperação aumentando a } \\
\text { eficiência do trabalho. }\end{array}$ \\
\hline 18 & $\begin{array}{l}\text { Avaliação Preliminar do } \\
\text { Programa de Humanização } \\
\text { no Pré-Natal e Nascimento } \\
\text { no Brasil. }\end{array}$ & $\begin{array}{l}\text { Serruya SJ, Lago TG, } \\
\text { Cecatti JG }\end{array}$ & $\begin{array}{l}\text { Análise documental e de } \\
\text { dados. }\end{array}$ & $\begin{array}{l}\text { Apesar de os indicadores de qualidade de } \\
\text { assistência mostrarem melhora de } 2001 \\
\text { para } 2002 \text {, há necessidade de permanentes } \\
\text { avaliações e intervenções afim de melhorar } \\
\text { a qualidade desta atenção, prioritariamente } \\
\text { nas regiões Norte e Nordeste. }\end{array}$ \\
\hline
\end{tabular}




\begin{tabular}{|c|c|c|c|c|}
\hline № & Título & Autores & Tipo de estudo & Recomendações/Conclusões \\
\hline 4 & $\begin{array}{l}\text { Sorologia anti-HIV e } \\
\text { aconselhamento quanto ao } \\
\text { pré-teste em gestantes na } \\
\text { região noroeste do Paraná, } \\
\text { Brasil. }\end{array}$ & $\begin{array}{l}\text { Misuta NM, Soares } \\
\text { DA, Souza RKT, } \\
\text { Matsuo T, Andrade } \\
\text { SM }\end{array}$ & Estudo transversal. & $\begin{array}{l}\text { A cobertura do teste anti-HIV durante a } \\
\text { gestação foi satisfatória. Porém, a baixa } \\
\text { frequência do aconselhamento e o atraso } \\
\text { no recebimento do resultado dos exames } \\
\text { são indicativos de problemas na atenção } \\
\text { pré-natal. }\end{array}$ \\
\hline 19 & $\begin{array}{l}\text { Aconselhamento sobre o } \\
\text { teste rápido anti-HIV em } \\
\text { parturientes }\end{array}$ & $\begin{array}{l}\text { Passos SCS, Oliveira } \\
\text { MIC, Júnior Saint Clair } \\
\text { SG, Silva KS }\end{array}$ & Estudo transversal. & $\begin{array}{l}\text { As mulheres em estado de vulnerabilidade } \\
\text { não foram alvo de aconselhamento, e } \\
\text { apenas as consultas pré-natais mostraram- } \\
\text { se um fator de proteção contra o não } \\
\text { aconselhamento. Sugere-se que o teste } \\
\text { rápido anti-HIV, vem sendo realizado sem o } \\
\text { consentimento das mulheres. }\end{array}$ \\
\hline 20 & $\begin{array}{l}\text { Frequência de testagem } \\
\text { rápida para o HIV durante } \\
\text { a admissão para o parto } \\
\text { em puérperas no Instituto } \\
\text { Materno Infantil Prof. } \\
\text { Fernando Figueira, IMIP }\end{array}$ & $\begin{array}{l}\text { Morimura MCR, } \\
\text { Mendes MDC, de } \\
\text { Souza Al, de Alencar } \\
\text { LCA }\end{array}$ & Estudo descritivo. & $\begin{array}{l}\text { A prevalência de testagem anti-HIV no pré- } \\
\text { natal foi satisfatória porém o recebimento } \\
\text { do resultado, bem como o aconselhamento } \\
\text { pós-teste, ficou aquém do recomendado. }\end{array}$ \\
\hline 21 & $\begin{array}{l}\text { Assistência pré-natal no } \\
\text { Brasil. Cad. Saúde Pública }\end{array}$ & $\begin{array}{l}\text { Viellas EF, Domingues } \\
\text { RMSM, Dias MAB, } \\
\text { Gama SGN , Theme } \\
\text { FMM, Costa JV et al }\end{array}$ & $\begin{array}{l}\text { Entrevista estruturada e } \\
\text { pesquisa ex-post facto. }\end{array}$ & $\begin{array}{l}58,7 \% \text { das entrevistadas foram orientadas } \\
\text { sobre a maternidade de referência, e } 16,2 \% \\
\text { procuraram mais de um serviço para a } \\
\text { admissão para o parto. Desafios persistem } \\
\text { para a redução de desfechos desfavoráveis. }\end{array}$ \\
\hline 22 & $\begin{array}{l}\text { Acesso à assistência pré- } \\
\text { natal no Brasil: análise dos } \\
\text { dados da Pesquisa Nacional } \\
\text { de Saúde }\end{array}$ & $\begin{array}{l}\text { Silva Nunes AD, } \\
\text { Amador AE, Dantas } \\
\text { APDQM, Azevedo UN } \\
\text { \& Barbosa IR }\end{array}$ & $\begin{array}{l}\text { Estudo transversal com } \\
\text { análise descritiva. }\end{array}$ & $\begin{array}{l}\text { Observou-se que, apesar de elevada } \\
\text { cobertura da assistência pré-natal no Brasil, } \\
\text { os indicadores mostram que ainda há } \\
\text { inadequações no acesso ao serviço. }\end{array}$ \\
\hline 23 & $\begin{array}{l}\text { Socio-economic and } \\
\text { ethnic group inequities in } \\
\text { antenatal care quality in the } \\
\text { public and private sector in } \\
\text { Brazil }\end{array}$ & $\begin{array}{l}\text { Victora CG, } \\
\text { Matijasevich A, } \\
\text { Silveira MF, Santos IS, } \\
\text { Barros AJD, Barros FC. }\end{array}$ & $\begin{array}{l}\text { Coleta de dados de base } \\
\text { bibliográfica. }\end{array}$ & $\begin{array}{l}\text { Há uma necessidade de monitoramento } \\
\text { regular dos atendimentos pré-natais e } \\
\text { qualidade dos cuidados com uma lente } \\
\text { de equidade, a fim de avaliar como } \\
\text { os diferentes grupos sociais estão se } \\
\text { beneficiando do progresso nos cuidados de } \\
\text { saúde. }\end{array}$ \\
\hline 24 & $\begin{array}{l}\text { Avaliação do pré-natal } \\
\text { quanto à detecção de } \\
\text { sífilis e HIV em gestantes } \\
\text { atendidas em uma área } \\
\text { rural do estado do Pará, } \\
\text { Brasil }\end{array}$ & $\begin{array}{l}\text { Araújo EC, Monte } \\
\text { Paula CB, Haber ANCA }\end{array}$ & $\begin{array}{l}\text { Estudo retrospectivo, } \\
\text { observacional e transversal. }\end{array}$ & $\begin{array}{l}\text { Observou-se que baixa escolaridade, falta } \\
\text { de trabalho remunerado, multiparidade, } \\
\text { baixa adesão aos exames de sífilis e de } \\
\text { HIV durante a gestação são relacionados } \\
\text { a falhas e baixa adesão ao pré-natal. } \\
\text { Devendo-se melhorar a qualidade do pré- } \\
\text { natal na realização desses exames, visando } \\
\text { à prevenção da transmissão vertical dessas } \\
\text { doenças. }\end{array}$ \\
\hline 25 & $\begin{array}{l}\text { Aconselhamento na } \\
\text { testagem anti-HIV no ciclo } \\
\text { gravídico-puerperal: o olhar } \\
\text { da integralidade. }\end{array}$ & $\begin{array}{l}\text { Carneiro AJS \& Coelho } \\
\text { EAC }\end{array}$ & $\begin{array}{l}\text { Estudo } \\
\text { qualitativo. }\end{array}$ & $\begin{array}{l}\text { A testagem anti-HIV foi incorporada à prática } \\
\text { clínica sem aconselhamento em nenhuma } \\
\text { das etapas da atenção ao ciclo gravídico- } \\
\text { puerperal. As relações entre profissionais } \\
\text { e usuárias refletem mecanismos de poder } \\
\text { que comprometem a autonomia das } \\
\text { mulheres. }\end{array}$ \\
\hline 26 & $\begin{array}{l}\text { Avaliação da qualidade do } \\
\text { rastreamento de HIV/aids } \\
\text { e sífilis na assistência pré- } \\
\text { natal. }\end{array}$ & Lima BGC et al & $\begin{array}{l}\text { Estudo de } \\
\text { transversal. }\end{array}$ & $\begin{array}{l}\text { Mulherescommelhor nívelde rendativeram } \\
\text { maior chance de iniciar precocemente a } \\
\text { APN, realizar maior número de consultas } \\
\text { e fazer os exames de rastreamento do HIV } \\
\text { e da sífilis ainda no } 1^{\circ} \text { trimestre, conforme } \\
\text { recomendado ao contrário das mulheres } \\
\text { em estado de vulnerabilidade. }\end{array}$ \\
\hline
\end{tabular}




\begin{tabular}{|c|c|c|c|c|}
\hline № & Título & Autores & Tipo de estudo & Recomendações/Conclusões \\
\hline 27 & $\begin{array}{l}\text { Gênero, estigma e saúde: } \\
\text { reflexões a partir da } \\
\text { prostituição, do aborto e do } \\
\text { HIV/Aids entre mulheres. }\end{array}$ & $\begin{array}{l}\text { Villela WV \& Monteiro } \\
\text { S }\end{array}$ & $\begin{array}{l}\text { Revisão narrativa } \\
\text { literatura. }\end{array}$ & $\begin{array}{l}\text { Ações no âmbito da gestão, na esfera } \\
\text { legislativa e em outros setores que } \\
\text { interferem na saúde, ao lado de práticas } \\
\text { cotidianas dos serviços de saúde contribuir } \\
\text { para ampliar seu acesso através do } \\
\text { reconhecimento da autonomia e direitos } \\
\text { da mulher. }\end{array}$ \\
\hline 28 & $\begin{array}{l}\text { Interdisciplinary action in } \\
\text { counseling for pregnant } \\
\text { women with HIV/Aids }\end{array}$ & $\begin{array}{l}\text { Araújo MAL, Reis } \\
\text { CBS, Andrade RFV, } \\
\text { Adriano LL, Silva RM } \\
\text { \& Gondim, APS }\end{array}$ & Pesquisa qualitativa. & $\begin{array}{l}\text { Identificou-se que as ações do } \\
\text { aconselhamento não atendiam a todos os } \\
\text { critérios recomendados pelo Ministério da } \\
\text { Saúde. }\end{array}$ \\
\hline 29 & $\begin{array}{l}\text { Cuidado de enfermagem } \\
\text { diante da prevenção da } \\
\text { transmissão vertical do HIV. }\end{array}$ & $\begin{array}{l}\text { Silva CRH, Rosendo } \\
\text { SRA \& Medeiros SM }\end{array}$ & Revisão integrativa. & $\begin{array}{l}\text { O enfermeiro deve conhecer os ângulos } \\
\text { dessa realidade para atuar com com maior } \\
\text { resolutividade e qualidade na prevenção da } \\
\text { transmissão vertical do HIV. }\end{array}$ \\
\hline
\end{tabular}

\section{DISCUSSÃO}

Em estudo transversal visando investigar os fatores associados ao diagnóstico de HIV, cujo resultado era ignorado pelas pacientes submetidas ao pré-teste, realizado em cinco hospitais públicos do Rio de Janeiro, todas as mulheres que realizaram o teste rápido anti-HIV por ocasião do parto, internadas em regime de alojamento conjunto, observou-se que, mesmo quando as pacientes realizavam o teste, muitas dessas mulheres não sabiam o resultado final de seus exames. Há uma perda de todo o trabalho realizado no pré-teste, pois sabe-se que o pré-teste é procedido de pós-teste com aconselhamento, em que ocorre uma discussão sobre o resultado do exame com o profissional de saúde, o resultado do exame deve ser entregue em mão ao paciente e anotado na carteirinha da gestante ${ }^{13}$.

Reiterando os dados do estudo anterior, Oliveira et al ${ }^{14}$ apontaram que não houve concordância em mais de um terço das informações documentadas referentes à submissão ao teste rápido anti-HIV, sugerindo registros incompletos e falhas no aconselhamento, o que contraria preconização do ministério da saúde que determina que as mulheres antes de serem submetidas ao pré-teste sejam consultadas a respeito de sua submissão ao exame, ignorando sua autonomia e a orientação sobre as ações de prevenção do HIV e da transmissão vertical ${ }^{15}$.

Outro grande problema diz respeito à capacitação; 13 enfermeiros entrevistados em estudo realizado na Paraíba, em 2017, revelaram que o tempo da capacitação não foi suficiente para deixar o profissional preparado para lidar com as outras etapas envolvidas no processo de testagem, como o aconselhamento e o encaminhamento do paciente com um resultado positivo para o HIV 16 . Não se deve restringir o atendimento dos usuários com HIV aos centros especializados, pois é uma situação que pode levar à descontinuidade da assistência para as pessoas com o vírus. A capacitação para lidar com a subjetividade do processo saúde/doença deve estar presente nos profissionais dos centros de saúde inseridos na comunidade à qual pertence o portador e este deve receber o apoio de outras categorias profissionais, pois por meio do trabalho em equipe, observa-se maior qualidade na prestação do atendimento ao portador ${ }^{17}$.

Em estudo realizado para avaliar a implantação do programa de humanização do pré-natal e do nascimento no Brasil, revela que a maioria das mulheres recebeu assistência desarticulada e parcial, e menos de $25 \%$ das mulheres tiveram seis ou mais consultas ${ }^{18}$.

Em estudo realizado no noroeste do Paraná, foi observado que a cobertura do teste anti-HIV durante a gestação foi satisfatória; porém, a baixa frequência do aconselhamento e o atraso no recebimento do resultado dos exames são indicativos de problemas na atenção pré-natal ${ }^{4}$. No entanto, em estudo realizado no Rio de Janeiro em $2013^{19}$ observou-se que apenas um quarto das parturientes foram aconselhadas e mais da metade sequer recebeu explicação sobre o porquê da realização do teste rápido anti-HIV, indicando que o teste vem sendo realizado sem consentimento das mulheres e de modo imperativo. Em Recife, foi encontrada uma prevalência de aconselhamento para o teste rápido anti-HIV por ocasião do parto ainda mais desfavorável: $7,7 \%{ }^{20}$.

De acordo com estudos $21,22,23$, o Brasil apresenta alta cobertura do pré-natal com pelo menos uma consulta, alcançando níveis superiores a $90 \%$, independentemente da região geográfica ou características das mães. No entanto, a ausência de pré-natal e a realização de pré-natal com menos de seis consultas foram fatores associados com diagnóstico tardio de HIV ${ }^{24}$. Devido à falta da integralidade do aconselhamento, pois muitas vezes só ocorre o pedido de exame, é importante os testes rápidos disponíveis em todas as unidades, e realizados no momento da abertura do pré-natal, não perdendo, assim, a chance de orientar sobre o exame a ser realizado, sua importância e já discutir o resultado ${ }^{25}$. Observa-se que o teste rápido não é um teste cotidiano; no momento do pré-natal, ocorre um apelo em torno da possibilidade da transmissão vertical do HIV que impulsiona a decisão de se submeter ao teste, sendo 
que esta é uma demanda do serviço, e não uma necessidade posta pela própria mulher com referência a possíveis riscos cotidianos, como o trabalho com sexo ${ }^{26}$. Sendo assim, um percentual considerável de mulheres acessa o teste via prénatal, como se esse fosse um procedimento próprio desse período e de proteção para a criança, não se revertendo em regularidade da testagem após o período gestacional, havendo, portanto, um entendimento de muitos polos de saúde que o teste rápido e o aconselhamento subsequente são apenas parte da rotina de cuidados na gestação. A dificuldade na testagem e no aconselhamento, como parte da vida cotidiana das mulheres com comportamento sexual de risco constitui uma vulnerabilidade ao HIV relacionadas com a invisibilidade da sexualidade feminina ${ }^{27}$.

Em outro estudo realizado no Amparo Maternal (AM), localizada na região Sudoeste do município de São Paulo, quando questionadas em relação a quem deveria colher os exames anti-HIV não sabiam que todos devem colher; nota-se que, durante a entrevista do pré-natal, não foi comunicado que o exame é solicitado a todas as gestantes que iniciam o pré-natal, lembrando que o exame não é obrigatório, e claro, que todas as pessoas devem colher o material para exame, independente do número de parceiros ou de opção sexual ${ }^{28}$.

O aconselhamento deve esclarecer aos pacientes sobre as formas de transmissão, de prevenção e de tratamento da AIDS, criar possibilidade para o paciente compreender as situações de risco de infecção a que ele pode estar exposto, alertar para a necessidade de realizar medidas de autocuidado e de cuidado com seus contatos sexuais ${ }^{29}$.

\section{CONCLUSÕES}

A AIDS é uma doença sexualmente transmissível, e a heterossexualização e feminização nos faz refletir que todos estamos vulneráveis ao vírus. A existência do teste rápido nas unidades favorece o diagnóstico precoce para as gestantes, agilizando o processo de encaminhamento e tratamento delas, quando o resultado é positivo. Da mesma forma, quando negativo o resultado do exame, o profissional de saúde deve atuar no aconselhamento correto incentivando o uso do preservativo.

O profissional da saúde deve estar capacitado e livre das barreiras do preconceito e do pré-julgamento para que consiga, por meio da educação em saúde, alcançar o seu maior objetivo: a confiança do paciente.

Além do teste, é realizado o aconselhamento pré e pós-teste, que é de suma importância para que a mulher saia do consultório com as dúvidas clarificadas, e não com mais dúvidas.

Muitas vezes, por falta de tempo, falta de habilidades, de interesse ou mesmo por desconhecimento, o enfermeiro deixa de aproveitar o momento da consulta para realizar o feedback com a gestante.

Os estudos analisados neste trabalho permitem concluir que é necessário agilizar o diagnóstico, mas também fornecer as condições e orientações necessárias para que o atendimento seja integral, e claro, fornecer subsídios para a ampliação da cobertura do teste rápido.

\section{REFERÊNCIAS}

1. Ministério da Saúde [BR]. Secretaria de Vigilância em Saúde. Programa Nacional de DST e AIDS. Bol epidemiológico AIDS e DST [Internet]. 2017 [acesso 2018 Aug 29] ; 5(3): 1- 84. Disponível em: http://www.saude.rs.gov.br/upload/ arquivos/carga20180508/11140851-boletim-2017.pdf.

2. Ministério da Saúde [BR]. Secretaria de Vigilância em Saúde. Programa Nacional de DST e AIDS. Bol epidemiológico AIDS e DST [Internet]. 2018 [acesso 2018 Aug 27]; 5(1):1-53. Disponível em: http://www.aids.gov.br/es/ node/65093.

3. Araújo MA, Vieira NF, Silva RM. HIV-testing for pregnant women in a Family Health Unit in Fortaleza, Ceará. Cien Saude Colet. 2008 Nov-Dec;13(6):1899-906. PubMed PMID: 18833367.

4. Misuta NM, Soares DA, Souza RKT, Matsuo T, Andrade SM. HIV serology and pretest counseling among pregnant women in the northwest region of Paraná State, Brazil. Rev. Bras. Saúde Matern Infant. 2008 Jan-Mar; 8(2):197-205. doi: http://dx.doi.org/10.1590/S1519-38292008000200007.

5. Soares ML, Oliveira MI, Fonseca VM, Brito AS, Silva KS. Predictors of unawareness of HIV serostatus among women submitted to the rapid HIV test at admittance for delivery. Cienc Saude Colet. 2013 May;18(5):1313-20. PubMed PMID: 23670459.

6. Lima SDS, Silva LCS, Santos MV, Martins JP, Oliveira MC, Brasileiro ME. HIV na gestação: pré-natal, parto e puerpério. Ciência \& Saúde. 2017 Jan-Mar; 10(1), 56-61. doi: http://dx.doi.org/10.15448/1983-652X.2017.1.22695.

7. Fonseca $\mathrm{PL}$, Iriart JAB. STD/Aids counseling for pregnant women who underwent the anti-HIV test on admission for delivery: the meanings of practice. Interface (Botucatu). 2012 Abr-Jun;16(41):395-407. doi: http://dx.doi. org/10.1590/S1414-32832012000200009.
8. Praça NS, Barrancos JT. HIV screening counseling in antenatal care: Perception of postpartum women. Rev Gaucha Enferm. 2007; 28(1):106-16.

9. Pereira BS, Costa MCO, Amaral MTR, Costa HS, Silva CAL, Sampaio VS. Fatores associados à infecção pelo HIV/AIDS entre adolescentes e adultos jovens matriculados em Centro de Testagem e Aconselhamento no Estado da Bahia, Brasil. Ciênc. saúde coletiva [Internet]. Mar 2014 [acesso 2018 Aug 30]; 19( 3 ): 747-758. doi: http://dx.doi.org/10.1590/1413-81232014193.16042013.

10. Nishimoto TM, Eluf J Neto, Rozman MA. Mother-to-child transmission of human immunodeficiency virus (HIV-I): evaluation of control measures in the city of Santos. Rev Assoc Med Bras. 2005 Jan-Fev; 51(1):54-60. doi: http:// dx.doi.org/10.1590/S0104-42302005000100021.

11. Silva SA \& Cubas MR. Saúde Coletiva: Linhas de Cuidado e Consulta de Enfermagem. Rio de Janeiro: Elsevier Brasil; 2012.

12. Mendes KDS, Silveira RCCP, Galvão CM. Revisão integrativa: método de pesquisa para a incorporação de evidências na saúde e na enfermagem. Texto contexto - enferm. 2008 Out-Dez; 17(4): 758-764. doi: http://dx.doi. org/10.1590/S0104-07072008000400018>.

13. Ministério da Saúde [BR]. Secretaria de Atenção à Saúde Departamento de Atenção Básica Atenção ao pré-natal de baixo risco. Série A. Normas e Manuais Técnicos Cadernos de Atenção Básica. Brasília: Ministério da Saúde; 2012. n 32.

14. Oliveira MIC, Silva KS, Gomes DM. Fatores associados à submissão ao teste rápido anti-HIV na assistência ao parto. Ciênc. Saúde col [Internet]. 2018 Fev [acesso 2018 Aug 25] ; 23( 2 ): 575-584. Disponível em: http://www.scielo. br/scielo.php?script=sci_abstract \&pid=S1413-81232018000200575\&lng=en\&n $\mathrm{rm}=\mathrm{iso} \& \mathrm{t} \operatorname{lng}=\mathrm{pt}$. 
15. Costa GR, Chein MB, Gama ME, Coelho LS, da Costa AS, Cunha CL, Brito LM. Caracterização da cobertura do pré-natal no Estado do Maranhão, Brasil. Rev Bras Enferm. 2010 Nov-Dec; 63(6):1005-9.

16. Silva TS, Nogueira CV, Rosendo RAS. Cartografia da implementação do teste rápido anti-HIV na Estratégia Saúde da Família: perspectiva de enfermeiros. Esc. Anna Nery. 2017;21(4):1-8

17. Pereira RCA, Rivera FJU, Artmann E. O trabalho multiprofissional na Estratégia Saúde da Família: estudo sobre modalidades de equipes. Interface (Botucatu). 2013 Abr-Jun; 17(45):327- 40. doi: http://dx.doi.org/10.1590/ S1414-32832013005000006.

18. Serruya SJ, Lago TG, Cecatti JG. Avaliação Preliminar do Programa de Humanização no Pré-Natal e Nascimento no Brasil. Rev Ginecol Obstet. 2004 Ago; 26(7): 517-25. doi: http://dx.doi.org/10.1590/S0100-72032004000700003.

19. Passos SCS, Oliveira MIC, Júnior Saint Clair SG, Silva KS. Aconselhamento sobre o teste rápido anti-HIV em parturientes. Rev. bras. epidemiol [Internet]. 2013 Jun [acesso 2018 Ago 29]; 16(2): 278-287. doi: http://dx.doi.org/10.1590/ S1415-790X2013000200005.

20. Morimura MCR, Mendes MDC, de Souza Al, de Alencar LCA. Freqüência de testagem rápida para o HIV durante a admissão para o parto em puérperas no Instituto Materno Infantil Prof. Fernando Figueira, IMIP. Rev Bras Saúde Mater Infant. 2006; 6 (Supl 1): 69-76. doi: http://dx.doi.org/10.1590/S1519 38292006000500010

21. Viellas EF, Domingues RMSM, Dias MAB, Gama SGN, Theme FMM, Costa JV et al . Assistência pré-natal no Brasil. Cad. Saúde Pública. 2014 [acesso 2018 Aug 30]; 30 (Suppl 1): S85-S100. Disponível em: http://www.scielo.br/scielo. php?script=sci_arttext\&pid=S0102-311X2014001300016.

22. Nunes ADS, Amador AE, Dantas APDQM, Azevedo UM, Barbosa IR. Acesso à assistência pré-natal no Brasil: análise dos dados da Pesquisa Nacional de Saúde. Rev Bras Promoç Saúde. 2017 Jul-Set;30(3). doi: http://dx.doi. org/10.5020/18061230.2017.6158.

23. Victora CG, Matijasevich A, Silveira MF, Santos IS, Barros AJD, Barros FC. Socio-economic and ethnic group inequities in antenatal care quality in the public and private sector in Brazil. Health Policy Plan. 2010 Jul; 25(4):253-61. doi: 10.1093/heapol/czp065. PubMEd PMID: 20123940.

24. Araújo EC, Monte Paula CB, Haber ANCA. Avaliação do pré-natal quanto à detecção de sífilis e HIV em gestantes atendidas em uma área rural do estado do Pará, Brasil. Rev Pan-Amaz Saude. 2018 Mar [acesso 2018 Ago 30]; 9(1): 33-39. Disponível em: http://scielo.iec.gov.br/scielo.php?script=sci_ arttext\&pid=S2176-62232018000100033.

25. Carneiro AJS, Coelho EAC. Aconselhamento na testagem anti-HIV no ciclo gravídico-puerperal: o olhar da integralidade. Ciênc. Saúde col. 2010 Jun; 15( Suppl 1 ): 1216-1226. doi: http://dx.doi.org/10.1590/S141381232010000700031

26. Lima BGC, Costa MCN, Dourado MIC. Avaliação da qualidade do rastreamento de HIV/aids e sífilis na assistência pré-natal. Epidemiol. Serv. Saúde. 2008 AbrJun;17(2):125-127.

27. Villela WV, Monteiro S. Gênero, estigma e saúde: reflexões a partir da prostituição, do aborto e do HIV/Aids entre mulheres. Epidemiol. Serv. Saúde. 2015 Jul-Set; 24 (3): 531-540.

28. Araújo MAL, Reis CBS, Andrade RFV, Adriano LL, Silva RM, Gondim, APS. Interdisciplinary action in counseling for pregnant women with HIV/Aids/ Ação interdisciplinar no aconselhamento para gestantes com HIV/Aids/Acción interdisciplinar en el consejo para mujeres embarazadas con HIV/Sida. Rev. Enferm UFPI, 2014 Jul-Set; 3(3), 80-7. doi: https://doi.org/10.26694/reufpi. v3i3.2124.

29. Silva CRH, Rosendo SRA, Medeiros SM. Cuidado de enfermagem diante da prevenção da transmissão vertical do HIV. Rev. Pesq. Cui. Fundam. Onl. 2015; 7(1): 2147-2158.

\section{Como citar este artigo/How to cite this article:}

Previati SM, Vieira DM, Barbieri M. A importância do aconselhamento no exame rápido de HIV em gestantes durante o pré-natal. J Health Biol Sci. 2018 Jan-Mar; 7(1):75-81. 